\title{
Simulação Computacional da Estabilização de Sistemas Subcríticos segundo o Modelo Unidimensional de Difusão de Nêutrons Monoenergéticos
}

\author{
Odair P. Silva, Hermes A. Filho, Ricardo C. Barros, \\ Programa de Pós-Graduação em Modelagem Computacional, IPRJ, UERJ, \\ Rua Bonfim, 25 - Vila Amélia - Cep 28.625-570, Nova Friburgo, RJ \\ E-mail: opsilva@iprj.uerj.br, halves@iprj.uerj.br, rcbarros@pq.cnpq.br.
}

\begin{abstract}
Resumo: Com o objetivo de descrever o perfil do fluxo escalar de nêutrons monoenergéticos em geometria unidimensional usando o modelo de difusão, desenvolvemos um método numérico espectronodal de difusão (END) em duas versões. A primeira versão considera problemas de autovalor, cujas soluções numéricas geradas pelo método END, sendo livres de erros de truncamento espacial, coincidem com a solução analítica dominante, afora erros da aritmética finita computacional. A segunda versão considera problemas de fonte fixa, onde a fonte é obtida analiticamente a partir da reconstrução espacial do fluxo escalar gerado pelo método END para problemas de autovalor. Neste caso também são obtidas soluções livres de erros de truncamento espacial. Um código computacional que implementa o método END foi desenvolvido em linguagem de programação C\# (C Sharp), a fim de gerar as soluções numéricas. A titulo de ilustração, são apresentadas soluções numéricas, geradas através deste código.
\end{abstract}

Palavras-chave: Difusão de nêutrons, Método espectronodal, Modelagem computacional

\section{1 - Introdução}

No contexto dos reatores nucleares do tipo ADS (Accelerator Driven System) [3], que consistem em sistemas subcríticos estabilizados por fonte fixa estacionária de nêutrons, desenvolvemos uma modelagem computacional para a estabilização de sistemas subcríticos com fonte estacionária. Para tanto, utilizamos um modelo simplificado unidimensional de difusão de nêutrons monoernergéticos para o qual foi desenvolvido um método espectronodal (END) com duas possibilidades. Na primeira, consideramos o convencional cálculo de autovalor, para o qual o método END gera soluções numéricas absolutamente livres de erros de truncamento espacial. A outra possibilidade é através do cálculo de fonte fixa estacionária de nêutrons, e também neste caso, o método END gera soluções numéricas livres de erros de truncamento espacial. Observamos aqui que, neste trabalho, desenvolvemos o método END para cálculos que utilizam fonte fixa variável com a posição no domínio, como ocorre com a fonte de fissão. A primeira fonte fixa que investigamos, consistia de uma fonte de fissão gerada analiticamente a partir do perfil do fluxo crítico obtido pela reconstrução espacial da solução de malha grossa do problema de autovalor. Constatamos neste caso, que as soluções obtidas nas duas possibilidades coincidem, a menos de erros de arredondamento da aritmética finita computacional. Em continuidade com o experimento numérico, inserimos uma perturbação no sistema que o colocasse em um estado subcrítico (reatividade negativa) ou em um estado supercrítico (reatividade positiva) [4]. È sabido que, o caso que desperta maior interesse de estudo é o estado subcrítico; então, determinamos o perfil do fluxo escalar complementar que deve ser somado ao perfil subcrítico a fim de se gerar a distribuição crítica de nêutrons no sistema. Portanto, a fonte estacionária de nêutrons monoenergéticos que estabilizará o sistema subcrítico na situação crítica será dada pelo produto do fluxo complementar pela seção de choque macroscópica de fissão da zona material pertinente dividida pelo fator de multiplicação efetivo (k), que é definido como autovalor dominante. En- 
fatizamos neste ponto que esta fonte fixa, conforme determinada pelo fluxo complementar de nêutrons, é única. Qualquer outro perfil de fonte fixa maior ou menor que o perfil determinado pelo fluxo complementar estabilizará o sistema subcrítico numa distribuição nêutrons maior ou menor que o perfil crítico.

\section{2 - O Método Espectronodal de Difusão (END)}

A precisão dos resultados numéricos gerados pelo método END deve-se ao fato de as equações de diferenças possuirem parâmetros determinados de tal forma a preservarem a solução geral local no interior de cada nodo de discretização espacial.

Apresentamos agora, uma descrição resumida do método END para problemas de autovalor. Considerando uma grade de discretização espacial arbitrária, conforme encontra-se ilustrada na Figura 1, onde cada célula ou nodo $\tau_{j}$ tem espessura $h_{j}, j=1: \mathrm{J}$, com J definido como o número total de nodos da grade, escrevemos a equação da continuidade e a lei de Fick como

$$
\begin{aligned}
& \frac{d}{d x} J(x)+\Sigma_{a_{j}} \Phi(x)=\frac{\nu}{k} \Sigma_{f_{j}} \Phi(x) \\
& J(x)=-D_{j} \frac{d}{d x} \Phi(x), \quad x_{j} \leq x \leq x_{j+1},
\end{aligned}
$$

onde $x_{j}$ e $x_{j+1}$ são as interfaces do nodo $\tau_{j}$.

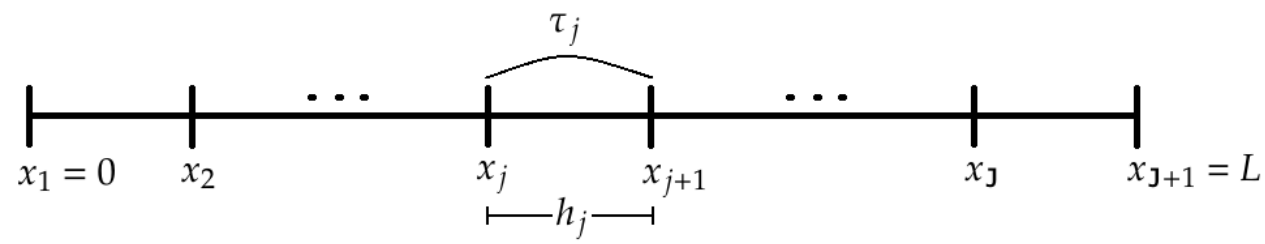

Figura 1: Grade de discretização espacial.

Podemos então apresentar a solução geral na forma

$$
\begin{aligned}
& \Phi(x)= \begin{cases}A_{1} \cos \left(\frac{x}{L_{j}(k)}\right)+A_{2} \operatorname{sen}\left(\frac{x}{L_{j}(k)}\right) & \text { caso complexo } \\
B_{1} \cosh \left(\frac{x}{L_{j}(k)}\right)+B_{2} \operatorname{senh}\left(\frac{x}{L_{j}(k)}\right) & \text { caso real }\end{cases} \\
& J(x)= \begin{cases}A_{1} \sqrt{D_{j}\left(\frac{\nu}{k} \Sigma_{f_{j}}-\Sigma_{a_{j}}\right)} \operatorname{sen}\left(\frac{x}{L_{j}(k)}\right) \\
-A_{2} \sqrt{D_{j}\left(\frac{\nu}{k} \Sigma_{f_{j}}-\Sigma_{a_{j}}\right)} \cos \left(\frac{x}{L_{j}(k)}\right) & \text { caso complexo } \\
-B_{1} \sqrt{D_{j}\left(\Sigma_{a_{j}}-\frac{\nu}{k} \Sigma_{f_{j}}\right)} \operatorname{senh}\left(\frac{x}{L_{j}(k)}\right) & \\
-B_{2} \sqrt{D_{j}\left(\Sigma_{a_{j}}-\frac{\nu}{k} \Sigma_{f_{j}}\right)} \cosh \left(\frac{x}{L_{j}(k)}\right) & \text { caso real }\end{cases}
\end{aligned}
$$

onde definimos o comprimento de difusão em meios multiplicativos como

$$
L_{j}(k)=\left\{\begin{array}{ll}
\sqrt{\frac{D_{j}}{\frac{\nu}{k} \Sigma_{f_{j}}-\Sigma_{a_{j}}}} & \text { se } \frac{\nu}{k} \Sigma_{f_{j}}>\Sigma_{a_{j}} \\
\sqrt{\frac{D_{j}}{\Sigma_{a_{j}}-\frac{\nu}{k} \Sigma_{f_{j}}}} & \text { caso contrário }
\end{array} .\right.
$$

Observamos que o caso complexo e caso real referem-se à natureza das raízes da equação característica de segundo grau, associada à equação diferencial ordinária de segunda ordem que se obtém ao substituir a Eq. (2) na Eq. (1).Agora, integramos as Eqs. (1) e (2) em $\tau_{j}$, aplicando o operador

$$
\frac{1}{h_{j}} \int_{x_{j}}^{x_{j+1}} \bullet d x
$$


Como resultado, obtemos as equações discretizadas de balanço espacial

$$
\begin{array}{r}
\frac{J_{j+1}-J_{j}}{h_{j}}+\Sigma_{a_{j}} \bar{\Phi}_{j}=\frac{\nu}{k} \Sigma_{f_{j}} \bar{\Phi}_{j} \\
\bar{J} i=-\frac{D_{j}}{h_{j}}\left(\Phi_{j+1}-\Phi_{j}\right)
\end{array}
$$

e escrevemos as equações auxiliares como

$$
\begin{aligned}
& \bar{\Phi}_{j}=\frac{\gamma_{j}}{2}\left(\Phi_{1+1}+\Phi_{j}\right) \\
& \bar{J}_{j}=\frac{\beta_{j}}{2}\left(J_{j+1}+J_{j}\right) .
\end{aligned}
$$

Aplicando o operador dado na Eq.(6) na Eq. (3) e substituindo na Eq. (9), obtemos duas equações. Procedimento análogo é seguido para a corrente. Partindo dessas equações, conseguimos concluir que

$$
\gamma_{j}=\beta_{j}= \begin{cases}\frac{2 L_{j}(k)}{h_{j}} \operatorname{tg}\left(\frac{h_{j}}{2 L_{j}(k)}\right) & \text { se } \frac{\nu}{k} \Sigma_{f_{j}}>\Sigma_{a_{j}} \\ \frac{2 L_{j}(k)}{h_{j}} \operatorname{tgh}\left(\frac{h_{j}}{2 L_{j}(k)}\right) & \text { caso contrário, }\end{cases}
$$

onde $L_{j}(k)$ está definido na Eq. (5). Para cada estimativa de $k$ no esquema iterativo de potência [1], calculamos $\gamma_{j}$ que depende dos parâmetros materiais e da espessura da região. As quantidades $\gamma_{j}$ preservam a solução geral analítica localizada em cada nodo de discretização espacial.

Em continuidade, apresentamos as equações para os fluxos no contorno exterior esquerdo, nas interfaces interiores e no contorno exterior direito. Essas equações foram determinadas a partir de manipulações algébricas utilizando as Eqs. (7), (8), (9), (10) e as condições de contorno generalizadas [5]. Assim, para o contorno exterior esquerdo utilizamos

$$
\left(\frac{D_{1}}{\gamma_{1} h_{1}}+\frac{1}{4} \gamma_{1} h_{1} \Sigma_{a_{1}}+\alpha_{0}\right) \Phi_{1}-\left(\frac{D_{1}}{\gamma_{1} h_{1}}-\frac{1}{4} \gamma_{1} h_{1} \Sigma_{a_{1}}\right) \Phi_{2}=\frac{\nu}{2 k} h_{1} \Sigma_{f_{1}} \bar{\Phi}_{1}
$$

para as interfaces interiores, temos

$$
\begin{aligned}
-\left(\frac{D_{j-1}}{\gamma_{j-1} h_{j-1}}-\frac{1}{4} \gamma_{j-1} h_{j-1} \Sigma_{a_{j-1}}\right) & \Phi_{j-1} \\
+\left(\frac{D_{j-1}}{\gamma_{j-1} h_{j-1}}+\frac{D_{j}}{\gamma_{j} h_{j}}+\frac{1}{4} \gamma_{j-1} h_{j-1} \Sigma_{a_{j-1}}+\frac{1}{4} \gamma_{j} h_{j} \Sigma_{a_{j}}\right) & \Phi_{j} \\
-\left(\frac{D_{j}}{\gamma_{j} h_{j}}-\frac{1}{4} \gamma_{j} h_{j} \Sigma_{a_{j}}\right) & \Phi_{j+1} \\
& =\frac{\nu}{2 k}\left(h_{j-1} \Sigma_{f_{j-1}} \bar{\Phi}_{j-1}+h_{j} \Sigma_{f_{j}} \bar{\Phi}_{j}\right)
\end{aligned}
$$

e para contorno exterior direito, usamos

$$
-\left(\frac{D_{\mathrm{J}}}{\gamma_{\mathrm{J}} h_{\mathrm{J}}}-\frac{1}{4} \gamma_{\mathrm{J}} h_{\mathrm{J}} \Sigma_{a_{I}}\right) \Phi_{\mathrm{J}}+\left(\frac{D_{\mathrm{J}}}{\gamma_{1} h_{\mathrm{J}}}+\frac{1}{4} \gamma_{\mathrm{J}} h_{\mathrm{J}} \Sigma_{a_{I}}+\alpha_{L}\right) \Phi_{\mathrm{J}+1}=\frac{\nu}{2 k} h_{\mathrm{J}} \Sigma_{f_{\mathrm{J}}} \bar{\Phi}_{\mathrm{J}} .
$$

Concluindo, acoplando a Eq. (12) com a Eq. (13) para $j=1: \mathrm{J}$ e a Eq. (14), teremos um sistema de $(\mathrm{J}+1)$ equações lineares e algébricas em $(\mathrm{J}+1)$ incógnitas $\Phi_{j}, j=1: \mathrm{J}$.

Com a convergência do método de potência, obtemos a solução dominante de malha grossa $\Phi_{j}, j=1$ : J. Como a solução numérica dominante normalizada pela potência nuclear ativa gerada $\Phi_{j}, j=1$ : J, é livre de erro de truncamento espacial, podemos usá-la como condição de fronteira na expressão da solução geral analítica local e determinarmos um par de constantes para cada nodo, cf. Eq. (3). Desta forma, podemos reconstruir $\Phi(x), 0 \leq x \leq L$, observando que 
é conveniente identificar $x_{j}=0$ e $x_{j+1}=h_{j}, j=1: \mathrm{J}$. Em outras palavras, a fronteira esquerda de cada nodo é sempre identificada como origem $(x=0)$ e, consequentemente, a fronteira direita deverá ser a espessura $h_{j}$ de cada nodo $\left(x=h_{j}\right)$. Portanto, escrevemos, cf. Eq. (3)

$$
\left\{\begin{array}{ll}
x_{j}=0 \Rightarrow A_{1}=B_{1}=\Phi_{j} ; & \text { caso complexo } \\
x_{j+1}=h_{j} \Rightarrow \frac{\Phi_{j+1}-\Phi_{j} \cos \left(\frac{h_{j}}{L_{j}(k)}\right)}{\operatorname{sen}\left(\frac{h_{j}}{L_{j}(k)}\right)} & \text { caso real } \\
B_{2}=\frac{\Phi_{j+1}-\Phi_{j} \cosh \left(\frac{h_{j}}{L_{j}(k)}\right)}{\operatorname{senh}\left(\frac{h_{j}}{L_{j}(k)}\right)} &
\end{array} .\right.
$$

Com o par de constantes determinado na Eq. (15) para cada região $j=1: \mathrm{J}$ do domínio, reconstruímos o perfil do fluxo escalar de nêutrons no interior de todo o domínio $0 \leq x \leq L$.

A partir deste ponto, passamos a descrever o método END para problemas de fonte fixa. Considerando as Eqs. (1) e (2), substituindo a fonte de fissão, termo à direita da Eq. (1), pela fonte fixa

$$
Q_{j}(x)= \begin{cases}\lambda \frac{\nu \Sigma_{f_{j}}}{k}\left(A_{1} \cos \left(\frac{x}{L_{j}(k)}\right)+A_{2} \operatorname{sen}\left(\frac{x}{L_{j}(k)}\right)\right) & \text { caso complexo, } \\ \lambda \frac{\nu \Sigma_{f_{j}}}{k}\left(B_{1} \cosh \left(\frac{x}{L_{j}(k)}\right)+B_{2} \operatorname{senh}\left(\frac{x}{L_{j}(k)}\right)\right) & \text { caso real }\end{cases}
$$

com $A_{1}, A_{2}, B_{1}$ e $B_{2}$ dados pela Eq. (15) e $\lambda=\frac{1}{1-\rho}$, sendo $\rho$ a reatividade [4], após algumas manipulações algébricas, podemos escrever

$$
\frac{d^{2}}{d x^{2}} \Phi(x)-\frac{1}{L_{j}^{2}} \Phi(x)= \begin{cases}-\lambda \frac{\nu \Sigma_{f_{j}}}{D_{j} k}\left(A_{1} \cos \left(\frac{x}{L_{j}(k)}\right)+A_{2} \operatorname{sen}\left(\frac{x}{L_{j}(k)}\right)\right) & \text { caso complexo, } \\ -\lambda \frac{\nu \Sigma_{f_{j}}}{D_{j} k}\left(B_{1} \cosh \left(\frac{x}{L_{j}(k)}\right)+B_{2} \operatorname{senh}\left(\frac{x}{L_{j}(k)}\right)\right) & \text { caso real, }\end{cases}
$$

onde $L_{j}=\sqrt{D_{j} / \Sigma_{a_{j}}}$ é definido como comprimento de difusão em meios não-multiplicativos. Observamos aqui, que se a reatividade for nula $(\rho=0$ e $\lambda=1)$, obtemos, de acordo com a Eq. (16), um perfil de fonte de fissão característico de sistema crítico. Entretanto, inserindo $\rho<0$, por exemplo, obtém-se $\lambda<1$ e, portanto, um perfil de fonte de fissão com menor intensidade, conforme Eq. (16). Esta fonte gerará uma distribuição de nêutrons inferior à distribuição crítica, cuja diferença ponto a ponto é definida neste trabalho como fluxo escalar complementar.

A solução geral local da Eq. (17) é dada por

$$
\Phi(x)=\Phi_{h}(x)+\Phi_{p}(x),
$$

i.e., a soma da solução homogênea com uma solução particular. A solução homogênea podemos escrever como

$$
\Phi_{h}(x)=C_{1} \cosh \left(\frac{x}{L_{j}}\right)+C_{2} \operatorname{senh}\left(\frac{x}{L_{j}}\right),
$$

e a solução particular, como

$$
\Phi_{p}(x)= \begin{cases}\lambda\left(A_{1} \cos \left(\frac{x}{L_{j}(k)}\right)+A_{2} \operatorname{sen}\left(\frac{x}{L_{j}(k)}\right)\right) & \text { caso complexo, } \\ \lambda\left(B_{1} \cosh \left(\frac{x}{L_{j}(k)}\right)+B_{2} \operatorname{senh}\left(\frac{x}{L_{j}(k)}\right)\right) & \text { caso real. }\end{cases}
$$

Para este caso, escrevemos as equações auxiliares como

$$
\begin{aligned}
& \bar{\Phi}_{j}=\frac{\gamma_{j}}{2}\left(\Phi_{j}+\Phi_{j+1}\right)+G_{f, i}, \\
& \bar{J}_{j}=\frac{\gamma_{j}}{2}\left(J_{j}+J_{j+1}\right)+G_{c, i} .
\end{aligned}
$$


Assumindo a solução geral dada pela Eq. (18), e aplicando a esta o operador dado na Eq.(6) obtemos uma equação que substituída na Eq. (21) nos permite concluir, a partir dos termos que envolvem os coeficientes $\left(C_{1}, C_{2}\right)$, que

$$
\gamma_{j}=\frac{2 L_{j}}{h_{j}} \operatorname{tgh}\left(\frac{h_{j}}{2 L_{j}}\right) .
$$

Portanto, $\gamma_{j}$ preserva a solução homogênea. Em prosseguimento, os termos que envolvem os coeficientes $\left(A_{1}, A_{2}\right)$ ou $\left(B_{1}, B_{2}\right)$, dependendo do caso, nos permitem determinar

$$
G_{f, i}=\left\{\begin{array}{l}
\lambda A_{1}\left(\frac{L_{j}(k)}{h_{j}} \operatorname{sen}\left(\frac{h_{j}}{L_{j}(k)}\right)-\frac{\gamma_{j}}{2}\left(1+\cos \left(\frac{h_{j}}{L_{j}(k)}\right)\right)\right)- \\
\lambda A_{2}\left(\frac{\gamma_{j}}{2} \operatorname{sen}\left(\frac{h_{j}}{L_{j}(k)}\right)-\frac{L_{j}(k)}{h_{j}}\left(1-\cos \left(\frac{h_{j}}{L_{j}(k)}\right)\right)\right) \quad \text { caso complexo, } \\
\lambda B_{1}\left(\frac{L_{j}(k)}{h_{j}} \operatorname{senh}\left(\frac{h_{j}}{L_{j}(k)}\right)-\frac{\gamma_{j}}{2}\left(1+\cosh \left(\frac{h_{j}}{L_{j}(k)}\right)\right)\right)- \\
\lambda B_{2}\left(\frac{\gamma_{j}}{2} \operatorname{senh}\left(\frac{h_{j}}{L_{j}(k)}\right)+\frac{L_{j}(k)}{h_{j}}\left(1-\cosh \left(\frac{h_{j}}{L_{j}(k)}\right)\right)\right) \quad \text { caso real, }
\end{array}\right.
$$

e

$$
G_{c, i}= \begin{cases}-D_{j} \lambda A_{1}\left(\frac{\gamma_{j}}{2 L_{j}(k)} \operatorname{sen}\left(\frac{h_{j}}{L_{j}(k)}\right)-\frac{1}{h_{j}}\left(1-\cos \left(\frac{h_{j}}{L_{j}(k)}\right)\right)\right)- & \\ D_{j} \lambda A_{2}\left(\frac{1}{h_{j}} \operatorname{sen}\left(\frac{h_{j}}{L_{j}(k)}\right)-\frac{\gamma_{j}}{2 L_{j}(k)}\left(1+\cos \left(\frac{h_{j}}{L_{j}(k)}\right)\right)\right) & \text { caso complexo, } \\ D_{j} \lambda B_{1}\left(\frac{\gamma_{j}}{2 L_{j}(k)} \operatorname{senh}\left(\frac{h_{j}}{L_{j}(k)}\right)+\frac{1}{h_{j}}\left(1-\cosh \left(\frac{h_{j}}{L_{j}(k)}\right)\right)\right)- & \\ D_{j} \lambda B_{2}\left(\frac{1}{h_{j}} \operatorname{senh}\left(\frac{h_{j}}{L_{j}(k)}\right)-\frac{\gamma_{j}}{2 L_{j}(k)}\left(1+\cosh \left(\frac{h_{j}}{L_{j}(k)}\right)\right)\right) \quad \text { caso real, }\end{cases}
$$

os parâmetros $G_{f, j}\left[\right.$ Eq. (24)] e $G_{c, j}[$ Eq. (25)] preservam a componente particular da solução geral.

Concluindo, a partir das equações auxiliares dadas pelas Eqs. (21) e (22), das condições de contorno generalizadas [5] e das equações de balanço espacial

$$
\begin{gathered}
\frac{J_{j+1}-J_{j}}{h_{j}}+\Sigma_{a_{j}} \bar{\Phi}_{j}=\overline{Q_{j}} \\
\bar{J}_{j}=-\frac{D_{j}}{h_{j}}\left(\Phi_{j+1}-\Phi_{j}\right),
\end{gathered}
$$

onde $\overline{Q_{j}}=\frac{1}{h_{j}} \int_{x_{j}}^{x_{j+1}} Q_{j}(x) d x$; determinamos equações que, assim como as Eqs. (12), (13) e (14), são utilizadas para calcular os fluxos escalares nos contornos exteriores esquerdo e direito e nas interfaces interiores. Então, para o contorno esquerdo, encontramos

$$
\left(\frac{D_{1}}{\gamma_{1} h_{1}}+\frac{\gamma_{1} h_{1} \Sigma_{a_{1}}}{4}+\alpha_{0}\right) \Phi_{1}-\left(\frac{D_{1}}{\gamma_{1} h_{1}}-\frac{\gamma_{1} h_{1} \Sigma_{a_{1}}}{4}\right) \Phi_{1}=\frac{h_{1} \overline{Q_{1}}}{2}-\frac{h_{1} \Sigma_{a_{1}}}{2} G_{f, 1}+\frac{1}{\gamma_{1}} G_{c, 1}
$$

para as interfaces interiores, obtemos

$$
\begin{aligned}
-\left(\frac{D_{j-1}}{\gamma_{j-1} h_{j-1}}-\frac{\gamma_{j-1} h_{j-1} \Sigma_{a_{j-1}}}{4}\right) & \Phi_{j-1} \\
+\left(\frac{D_{j-1}}{\gamma_{j-1} h_{j-1}}+\frac{D_{j}}{\gamma_{j} h_{j}}+\frac{\gamma_{j-1} h_{j-1} \Sigma_{a_{j-1}}}{4}+\frac{\gamma_{j} h_{j} \Sigma_{a_{j}}}{4}\right) & \Phi_{j} \\
-\left(\frac{D_{j}}{\gamma_{j} h_{j}}-\frac{\gamma_{j} h_{j} \Sigma_{a_{j}}}{4}\right) & \Phi_{j+1} \\
& =\frac{h_{j} \overline{Q_{j}}}{2}-\frac{h_{j} \Sigma_{a_{j}}}{2} G_{f, i}+\frac{1}{\gamma_{j}} G_{c, i} \\
& +\frac{h_{j-1} \overline{Q_{j-1}}}{2}-\frac{h_{j-1} \Sigma_{a_{j-1}}}{2} G_{f, i-1} \\
& -\frac{1}{\gamma_{j-1}} G_{c, i-1} .
\end{aligned}
$$


e por fim, para o contorno direito, temos

$$
-\left(\frac{D_{\mathrm{J}}}{\gamma_{\mathrm{J}} h_{\mathrm{J}}}-\frac{\gamma_{\mathrm{J}} h_{\mathrm{J}} \Sigma_{a_{\mathrm{J}}}}{4}\right) \Phi_{\mathrm{J}}+\left(\frac{D_{\mathrm{J}}}{\gamma_{\mathrm{J}} h_{\mathrm{J}}}-\frac{\gamma_{\mathrm{J}} h_{\mathrm{J}} \Sigma_{a_{\mathrm{J}}}}{4}+\alpha_{L}\right) \Phi_{\mathrm{J}+1}=\frac{h_{\mathrm{J}} \overline{Q_{\mathrm{J}}}}{2}-\frac{h_{\mathrm{J}} \Sigma_{a_{\mathrm{J}}}}{2} G_{f, \mathrm{~J}}-\frac{1}{\gamma_{\mathrm{J}}} G_{c, \mathrm{~J}},
$$

aqui também obtemos um sistema de $(\mathrm{J}+1)$ equações lineares e algébricas em $(\mathrm{J}+1)$ incógnitas $\Phi_{j}, j=1: \mathrm{J}$, como resultado do acoplamento das Eqs. (28) e (30) com a Eq. (29) para $j=1: \mathrm{J}$.

\section{3 - Resultados Numéricos}

Os parâmetros materiais de cada uma das regiões do problema-modelo estão listados na Tabela 1, e foram determinados pela média aritmética dos parâmetros dos dois grupos fornecidos por [2]. O problema-modelo considerado constitui-se de um domínio heterogêneo composto pelas dez regiões listadas na Tabela 1 , com condições de contorno tipo fluxo escalar nulo para $x=0$ cm e $x=384,758 \mathrm{~cm}$ e potência gerada de $1000 \mathrm{MW}$.

\begin{tabular}{ccccc}
\hline Região & $\mathrm{D}^{\mathrm{a}}$ & $\Sigma_{a}{ }^{\mathrm{b}}$ & $\nu \Sigma_{f}^{\mathrm{c}}$ & Largura da região $(\mathrm{cm})$ \\
\hline 1 & 0,89860 & 0,0054383 & 0,0 & 4,0 \\
2 & 1,20320 & 0,0329474 & 0,0466941 & 26,126 \\
3 & 1,19055 & 0,0349528 & 0,0479517 & 52,251 \\
4 & 1,16020 & 0,0352893 & 0,0476130 & 52,251 \\
5 & 1,11820 & 0,0352074 & 0,0468138 & 52,251 \\
6 & 1,05625 & 0,0349876 & 0,0455437 & 52,251 \\
7 & 0,96850 & 0,0346528 & 0,0437822 & 52,251 \\
8 & 0,89530 & 0,0345124 & 0,0427313 & 52,251 \\
9 & 0,87110 & 0,0348903 & 0,0451505 & 26,126 \\
10 & 0,69230 & 0,0182700 & 0,0 & 15,0 \\
\hline
\end{tabular}

${ }^{\text {a }}$ Coeficiente de difusão.

bSeção de choque macroscópica de absorção.

${ }^{c}$ Seção de choque macroscópica de fissão multiplicada pelo número médio de nêutrons gerados.

Tabela 1: Parâmetros físicos do problema-modelo

A Tabela 2 apresenta os resultados numéricos de um experimento considerando o problemamodelo que, supostamente, é constituído do combustível $P u^{239}$, onde foi inserida uma reatividade uniforme de $-0,30 \$$. Como efeito disto, a fonte fixa de fissão torna-se menor do que a fonte crítica. A primeira coluna da Tabela 2 lista as posições do domínio onde foram avaliados os fluxos escalares pelo método END de fonte fixa, na segunda coluna esses fluxos são exibidos. Na terceira coluna da Tabela 2 é listado o perfil do fluxo complementar que somando aos valores do fluxo subcrítico, mostrados segunda coluna, obtemos o perfil do fluxo crítico, exposto na quarta coluna da Tabela 2.

\section{4 - Conclusões}

O método END é livre de erros de truncamento espacial no sentido de que ele gera soluções numéricas coincidentes com os resultados gerados a partir da solução analítica dominante. Isto se deve ao fato de que as equações discretizadas do método END possuem parâmetros que preservam a solução geral local analítica no interior de cada região do domínio. Portanto, afora os erros de arredondamento da aritmética finita computacional, os resultados gerados pelo método END são "exatos". A modelagem matemática aqui apresentada nos permitiu implementar um algoritmo numérico num aplicativo computacional que determine analiticamente o perfil estacionário da fonte externa de nêutrons que estabilize um sistema subcrítico, como ilustra a Tabela 2. Esta contribuição encontra-se no contexto dos sistemas estabilizados por aceleradores, i.e., os Accelerator Driven Systems (ADS), que foram mencionados na introdução deste trabalho. 
Proceeding Series of the Brazilian Society of Applied and Computational Mathematics, Vol. 3, N. 1, 2015.

\begin{tabular}{cccc}
\hline Posição $^{\mathrm{a}}$ & Fluxo Subcrítico $^{\mathrm{b}}$ & Fluxo Complementar $^{\mathrm{c}}$ & Fluxo Crítico $^{\mathrm{d}}$ \\
\hline 4 & $9,60234 \mathrm{E}+17$ & $5,76140 \mathrm{E}+14$ & $9,60810 \mathrm{E}+17$ \\
30,126 & $4,67465 \mathrm{E}+18$ & $2,80479 \mathrm{E}+15$ & $4,67745 \mathrm{E}+18$ \\
82,377 & $5,27916 \mathrm{E}+18$ & $3,16750 \mathrm{E}+15$ & $5,28233 \mathrm{E}+18$ \\
134,628 & $2,38481 \mathrm{E}+18$ & $1,43089 \mathrm{E}+15$ & $2,38624 \mathrm{E}+18$ \\
186,879 & $6,16349 \mathrm{E}+17$ & $3,69810 \mathrm{E}+14$ & $6,16719 \mathrm{E}+17$ \\
239,130 & $8,91538 \mathrm{E}+16$ & $5,34923 \mathrm{E}+13$ & $8,92073 \mathrm{E}+16$ \\
291,381 & $6,93700 \mathrm{E}+15$ & $4,16220 \mathrm{E}+12$ & $6,94116 \mathrm{E}+15$ \\
343,632 & $4,00362 \mathrm{E}+14$ & $2,40217 \mathrm{E}+11$ & $4,00602 \mathrm{E}+14$ \\
369,758 & $6,96948 \mathrm{E}+13$ & $4,18168 \mathrm{E}+10$ & $6,97366 \mathrm{E}+13$ \\
\hline
\end{tabular}

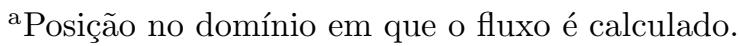

${ }^{b}$ Fluxo escalar de nêutrons calculado com o método END de fonte fixa.

${ }^{\mathrm{c}}$ Fluxo complementar calculado analiticamente.

${ }^{\mathrm{d}}$ Fluxo escalar de nêutrons obtido pela soma das colunas 2 e 3.

Tabela 2: Resultados numéricos do problema-modelo

É importante observar neste ponto que apesar de ter sido incluída reatividade negativa no problema-modelo considerado na seção anterior, a metodologia adequa-se igualmente a inserções de reatividades positivas.

Como trabalhos futuros, propomos a extensão da metodologia aqui descrita para modelos de difusão e de transporte de nêutrons na formulação multigrupo de energia. Fica também como sugestão estender a presente estratégia de modelagem computacional de estabilização de sistemas subcríticos com fontes estacionárias para geometrias multidimensionais.

\section{Referências}

[1] R. L. BURDEN and J. D. FAIRES. Numerical analysis. New York, Dover,USA, 8 edition, 1993.

[2] G. E. Fladmark. A method for the numerical solution of eigenvalue problems in diffusion theory. IAEA-SM-154/20, pages 497-507, 1972.

[3] L. García, J. Pérez, C. García, A. Escrivá, J. Rosales, and A. Abánades. Calculation of the packing fraction in a pebble-bed ads and redesigning of the transmutation advanced device for sustainable energy applications (tadsea). Nuclear Engineering and Design, (253):142-152, 2012 .

[4] J. R. LAMARSH. Introduction to nuclear reactor theory. Addison-Wesley Publishing Company, 1966.

[5] R. S. Mansur, F. P. Santos, H. Alves Filho, and R. C. Barros. Diffusion synthetic methods for computational modeling of one-speed slab-geometry transport problems with linearly anisotropic scattering. Progress in nuclear energy, Aceito para publicação em 2013. 